\title{
Équilibre dynamique d'une voile par une approche fluide structure
}

\author{
SÉbastien Mounoury ${ }^{1}$, Frédéric Hauville ${ }^{1, a}$, Yann Roux ${ }^{2}$ Et Jacques André Astolfi ${ }^{1}$ \\ 1 École Navale, Institut de Recherche de l'École Navale (IRENav EA 3634), Lanvéoc Poulmic, BP 600, 29240 Brest Armées, \\ France \\ ${ }^{2}$ Société K-EPSILON, 30 rue de Richelieu, 76087 Le Havre, France
}

Reçu le 14 janvier 2005, accepté le 13 octobre 2005

\begin{abstract}
Résumé - Le coût élevé des essais en bassin de carène associé aux performances accrues des moyens de calcul amène les architectes navals à utiliser de plus en plus les outils de simulations numériques pour la conception de voiliers. Ainsi, de nouveaux VPP (Velocity Prediction Programm) entièrement numériques se sont développés [Roux et al. Yacht performance prediction: Towards a numerical vpp, in : High Performance Yacht Design Conference, December 2002, Auckland]. Ces outils, basés sur la modélisation aéroélastique des voiles couplée à un modèle d'écoulement hydrodynamique avec surface libre autour de la carène, permettent la modélisation numérique d'un voilier en navigation réelle. L'étude présentée traite de la modélisation aéroélastique. Elle porte sur l'interaction d'une structure tridimensionnelle idéalement flexible avec un écoulement instationnaire. L'objectif est d'inclure la déformation et le déplacement du câble supportant la voile d'avant du voilier (l'étai) qui modifie le volume de la voile et donc le fonctionnement du voilier. Le problème aéroélastique couplé est résolu par une méthode itérative où les simulations élastiques et aérodynamiques se succèdent pour converger vers un équilibre statique. Nous introduisons plus particulièrement dans ce travail la résolution mécanique d'un câble soumis à un chargement tridimensionnel par une méthode variationnelle et son couplage avec la voile. Le comportement mécanique de la voile est modélisé par un système de réseau de fils [Le Maître, Contribution numérique à la résolution de problèmes d'interaction fluide structure, Thèse, Institut de mécanique INSA de Rouen, France, 1998] et l'écoulement aérodynamique par une méthode de surface portante associée à une méthode particulaire [Charvet et al., Numerical simulation of the flow over sails in real sailing conditions, J. Wind Eng. Ind. Aerod. Special Issue Sail Aerodynamics 63 (1996) 111-129]. Nous présentons les résultats d'un calcul aéroélastique d'une voile sur son étai soumis à un écoulement de fluide parfait dans lesquels l'influence d'un étai libre sur le comportement d'une voile est mise en évidence.
\end{abstract}

Mots clés : Interaction fluide structure / réseau de fils / méthode particulaire / structures souples / dynamique du voilier

\begin{abstract}
Dynamical balance of a yacht sail by a fluid structure approach. The high cost of the towing tank tests associated with the computer's progress leads naval architects to increasingly use numerical tools for sailing yacht design. Thus, new entirely numerical Velocity Prediction Programs are developed [Roux et al. Yacht performance prediction Towards a numerical vpp, in: High Performance Yacht Design Conference, December 2002, Auckland]. These tools, based on the aeroelastic model of the sails coupled to a model of hydrodynamic flow with free-surface around the hull, allow the numerical modelling of a sailing yacht in real navigation. The study presented the fluid-structure interaction model. It relates to the interaction of an ideally flexible three-dimensional structure with a non stationary flow. The objective of this study is to include the deformation and the displacement of the cable supporting the genoa (the stay) which modifies the volume of the sail and thus the behavior of the sailing yacht. The coupled aeroelastic problem is solved by an iterative method where elastic and aerodynamic simulations follow one another to converge towards a static balance. In particular, we introduce into this work the mechanical resolution of a cable subjected to a three-dimensional loading by a variational method and its coupling with the sail. The mechanical behavior of the sail is modelled by a string network system [Le Maître, Contribution numérique à la résolution de problèmes d'interaction fluide structure, Ph.D. Thesis, Institut de mécanique INSA de Rouen,
\end{abstract}

a Auteur correspondant : hauville@ecole-navale.fr 
France, 1998] and the aerodynamic model by a boundary element method for the lifting surface associated to a vortex element method for the wake [Charvet et al., Numerical simulation of the flow over sails in real sailing conditions, J. Wind Eng. Ind. Aerod. Special Issue Sail Aerodynamics 63 (1996) 111-129]. We show the results of an aeroelastic computation of a sail on its stay subjected to a tri-dimensional flow of inviscid fluid in which the influence of a free stay on the behavior of a sail is highlighted.

Key words: Fluid structure interaction / string network model / vortex element method / flexible structure / dynamics of the yacht sail

\section{Introduction}

La modélisation du comportement mécanique des voiles de bateau a déjà donné lieu à plusieurs travaux qui n'ont pas toujours conduit à publication, le secret étant souvent de mise. Le modèle de (1992) [4] décompose le comportement de la voile en une superposition de deux sous-modèles. Dans le premier, la voile est considérée parfaitement flexible et inextensible. Les solutions admissibles sont celles qui conservent les distances entre nœuds voisins et qui maximisent le travail des forces externes. Dans le deuxième, la déformation élastique de la voile est calculée par un modèle de coque mince. Les déplacements sont considérés comme infinitésimaux et les formulations en élasticité sont linéarisées. Cette méthode conduit à des résultats non réalistes lorsque les frontières de la voile sont libres et peuvent subir de grands déplacements, lorsque le module d'Young diminue et lorsque les surfaces sont planes ou quasi-planes. Muttin [5] a développé une approche du comportement d'une voile par une succession de déplacements infinitésimaux d'une membrane. Entre deux déplacements il résout le problème d'élasticité linéaire. Une déformation finie est ainsi calculée à partir de la série de déformation infinitésimale. Mais le modèle n'est valide que pour les approches incluant une déformation élastique, il n'est plus applicable pour les cas de figure où seules les déformations souples rentrent en jeu sans inclure de variation de l'énergie interne. Un modèle non-linéaire prenant en compte simultanément les déformations souples et élastiques est nécessaire, c'est ce que propose le modèle développé par Le Maître et al. [6]. Ce modèle approche la surface d'une voile par un réseau de fils constitué de deux familles de fils perpendiculaires. Il permet de prendre en compte à la fois les déformations élastiques et les grands déplacements que la voile subit du fait de sa très grande flexibilité. Néanmoins, ce modèle ne permet pas de traiter les voiles « moulées » type 3DL ou D4. Des travaux plus récents sur des codes membranaires ont permis de prendre en compte les non-linéarités géométriques liées aux déplacements des voiles et le comportement spécifique des tissus $[7,8]$.

Nous présentons dans ce travail l'optimisation d'un modèle d'interaction fluide structure [9] par la prise en compte de la déformation et du déplacement du câble supportant la voile d'avant : l'étai. En effet, le volume de la voile, d'une part, et la configuration du bord d'attaque de la voile, d'autre part, qui sont largement influencés par la flèche d'étai, modifient les performances du bateau.

La première partie est consacrée à la description du modèle de câble soumis à un chargement tridimensionnel et à sa validation sur le problème de la chaînette.
Dans la deuxième partie nous décrivons la modélisation numérique d'une voile et le couplage avec l'étai. La troisième partie reprend succinctement la description de la méthode fluide employée. Dans la dernière partie, l'influence d'un étai libre sur le comportement d'une voile, évoqué par Valette [7], est mis en évidence.

\section{Mécanique du fil et modélisation}

Les câbles et les fils sont des structures souples caractérisées par une section de dimension négligeable par rapport à la longueur. Ils ont la particularité de n'avoir comme efforts internes que des efforts de traction. Ils sont soumis à de grands déplacements, ce qui rend leur analyse mécanique fortement non-linéaire.

\subsection{Hypothèses}

Le modèle de fil, basé sur les travaux de Souza De Cursi [12], repose sur les hypothèses suivantes :

- Le fil est une structure unidimensionnelle qui est entièrement décrit par sa ligne moyenne.

- Le fil n'admet pas de déformation élastique transversale : la section du fil reste constante.

- Le fil ne peut supporter aucun moment de flexion ni de torsion : le fil est considéré comme une structure idéalement flexible.

- Le fil a un comportement unilatéral : il ne peut transmettre des efforts de compression (allongement négatif).

- Nous restons dans le domaine des petites déformations élastiques ce qui nous permet de reprendre les approximations faites dans la théorie de l'élasticité linéaire.

Les efforts internes se résument à des efforts de traction, ce qui simplifie l'expression du tenseur des contraintes. Ces tensions sont tangentes au fil en tout point (conservation du moment angulaire). Le fil est susceptible de subir de grands déplacements (appelés aussi déformations souples) ce qui empêche toutes approximations sur ceux-ci.

\subsection{Formulation du problème}

Nous considérons un câble caractérisé par sa longueur naturelle $L$, sa section $A$, son module d'Young $E$ et sa masse volumique $\rho$. Chaque point physique de la configuration de référence du câble est repéré par l'abscisse 
curviligne $s \in[0, L]$. Une liaison rotule est imposée aux extrémités $S_{1}$ et $S_{2}$ du câble. Le centre de cette liaison est fixe dans le repère absolu. À cette coordonnée lagrangienne $s$ est associée un vecteur position $\underline{\boldsymbol{r}}(s) \in \mathcal{R}^{3}$ permettant de décrire la configuration courante du câble. Un vecteur $\underline{\boldsymbol{t}}(s)$ tangent à la ligne moyenne en tout point $s$ est défini par :

$$
\underline{\boldsymbol{t}}(s)=\frac{\frac{\partial \underline{\boldsymbol{r}}(s)}{\partial s}}{\left\|\frac{\partial \underline{\boldsymbol{r}}(s)}{\partial s}\right\|}
$$

La configuration initiale arbitraire pour le problème fortement non-linéaire de la chaînette (catenaria) est un demi-cercle de longueur $L$ (pas de précontrainte) dans le plan $O x z\left(s_{1}(0,0,0), s_{2}\left(\frac{2 L}{\pi}, 0,0\right)\right)$. Le chargement extérieur considéré est le poids de la structure $\underline{\boldsymbol{P}}=$ $A L \rho g \underline{\mathbf{y}}$. La réponse de la structure pour l'équilibre se traduit par des déplacements $\underline{\boldsymbol{r}}(s)$ et des déformations élastiques $T(s) \underline{\boldsymbol{t}}(s)$. L'équation du mouvement exprimant l'équilibre dynamique du câble peut être posée comme suit :

$$
\frac{\mathrm{d} \underline{\boldsymbol{T}}(s)}{\mathrm{d} s}+A \rho(\underline{\boldsymbol{g}}-\underline{\ddot{\boldsymbol{r}}}(s))=\underline{\mathbf{0}}
$$

Le développement de l'expression de la tension fait apparaître dans cette équation l'inconnue du problème, le vecteur position $\underline{\boldsymbol{r}}(s)$. Afin de résoudre plus facilement l'équation (2), nous appliquons le principe des puissances virtuelles entre les deux extrémités du câble où $\boldsymbol{V}^{*}$ est une vitesse virtuelle :

$$
\int_{s_{1}}^{s_{2}}\left[\underline{\boldsymbol{T}}(s) \cdot \frac{\mathrm{d} \underline{\boldsymbol{V}^{*}}(s)}{\mathrm{d} s}-A \rho(\underline{\boldsymbol{g}}-\underline{\ddot{\boldsymbol{r}}}(s)) \cdot \underline{\boldsymbol{V}}^{*}(s)\right] \mathrm{d} s=\underline{\mathbf{0}}
$$

Ces tensions $\underline{\boldsymbol{T}}(s)$ sont dues aux efforts de cohésion du matériau. Elles apparaissent comme une réponse de la structure au chargement extérieur. En effet, sous la contrainte de ce dernier, la structure va se déformer de deux manières :

- Une déformation élastique, qui correspond ici à une modification de la longueur entre les points de la structure sur la ligne moyenne. C'est cette déformation qui va induire des forces internes.

- Une déformation souple qui n'est en fait qu'un déplacement de la structure par translation et rotation qui entraîne un changement d'orientation des forces internes sans en modifier leur intensité.

La loi de comportement du matériau permet de faire le lien entre les forces internes et les déformations pour déterminer la configuration géométrique :

$$
\underline{\boldsymbol{T}}(s)=A E(\alpha(s)-1) \underline{\boldsymbol{t}}(s) \geq 0
$$

où $\alpha(s)$ représente la dilatation longitudinale qui est le rapport entre la longueur d'arc courante et la longueur d'arc naturelle :

$$
\begin{aligned}
\alpha(s)=\left\|\frac{\mathrm{d} \underline{\boldsymbol{r}}(s)}{\mathrm{d} s}\right\| \text { si }\left\|\frac{\mathrm{d} \underline{\boldsymbol{r}}(s)}{\mathrm{d} s}\right\| \geq 1 \text { et } \\
\alpha(s)=1 \text { si }\left\|\frac{\mathrm{d} \underline{\boldsymbol{r}}(s)}{\mathrm{d} s}\right\|<1
\end{aligned}
$$

Finalement, l'équation à résoudre traduisant l'équilibre dynamique du câble est :

$$
\begin{aligned}
\int_{s 1}^{s 2}[A E(\alpha(s)-1) & \underline{\boldsymbol{t}}(s) \cdot \frac{\mathrm{d} \underline{\boldsymbol{V}^{*}}(s)}{\mathrm{d} s} \\
& \left.-A \rho(\underline{\boldsymbol{g}}-\underline{\ddot{\boldsymbol{r}}}(s)) \cdot \underline{\boldsymbol{V}}^{*}(s)\right] \mathrm{d} s=\underline{\mathbf{0}}
\end{aligned}
$$

\subsection{Analyse numérique}

L'équation (6), discrétisée à l'aide de la méthode des éléments finis, est résolue de manière approchée par une méthode itérative directe. Le câble est discrétisé en $N_{e}$ éléments linéaires à deux nœuds indicés $i \in\left[0, N_{n}=\right.$ $\left.N_{e}+1\right]$. La masse de l'élément linéaire est concentrée en ses nœuds. Les deux nœuds indicés $i$ et $i+1$ sont séparés par un élément câble sans masse indicé $i$, de longueur naturelle $l_{i}$, pouvant s'allonger par déformation élastique. Les éléments câbles peuvent avoir une libre rotation autour des nœuds (rotule). Sous forme discrétisée, l'équation (6) devient :

$$
\begin{aligned}
& \sum_{i=1}^{N_{n}-1} \underline{\boldsymbol{\Theta}}_{i} \cdot \underline{\boldsymbol{V}}_{i}^{*}=0 \quad \text { et } \\
& \underline{\boldsymbol{\Theta}}_{i}=\underline{\boldsymbol{P}}_{i}+A E\left(\alpha_{i-1}-1\right) \underline{\boldsymbol{t}}_{i-1}-A E\left(\alpha_{i}-1\right) \underline{\boldsymbol{t}}_{i}
\end{aligned}
$$

$\underline{\boldsymbol{\Theta}}_{i}$ représente la résultante des forces au nœud $i$ qui dépend de $\underline{\boldsymbol{r}}_{i}, \underline{\boldsymbol{r}}_{i-1}$ et $\underline{\boldsymbol{r}}_{i+1}$ et $\underline{\boldsymbol{P}}_{i}$ représente le chargement externe dans lequel on retranche l'accélération locale pour une analyse dynamique $\left(\underline{\boldsymbol{P}}_{i}=A l_{i} \rho\left(\underline{\boldsymbol{g}}-\underline{\ddot{\boldsymbol{x}}}_{i}\right)\right)$. Pour tous les nœuds excepté pour le nœud $i$, on pose $\underline{\boldsymbol{V}}_{i}^{*}=\underline{\mathbf{0}}$. Nous obtenons ainsi un système de $3\left(N_{n}-1\right)$ équations nonlinéaires résolu par une méthode itérative directe. Chaque composante du vecteur position du noud $i$ à l'itération $k+1, \underline{\boldsymbol{r}}_{i}^{k+1}$ est calculée de la manière suivante :

$$
\underline{\boldsymbol{r}}_{i}^{k+1}=\underline{\boldsymbol{r}}_{i}^{k}+\underline{\mathcal{O}}_{i}^{k} \zeta
$$

$\underline{\mathcal{O}}_{i}^{k}$ correspond à la résultante des forces au nœud $i$ à l'itération $k$ qui tend vers zéro à l'équilibre. $\zeta$ représente un coefficient de sous-relaxation qui permet de limiter les variations apportées aux vecteurs position et ainsi éviter la divergence. Ce coefficient est globalement inversement proportionnel au module d'Young E. Le critère de convergence porte sur la variation du résidu moyen calculé sur l'ensemble des nœuds entre $N$ itérations.

$$
\begin{aligned}
\text { Norm }^{k}-\text { Norm }^{k-N} \leq \varepsilon & \text { et } \\
& \operatorname{Norm}^{k}=\frac{1}{N_{n}} \sum_{i=1}^{N_{n}}\left\|\underline{\mathcal{O}}_{i}^{k}\right\|
\end{aligned}
$$

\subsection{Résultats numériques}

Le problème de la chaînette est appliqué sur un fil dont les caractéristiques sont proches de celles d'un étai d'un 


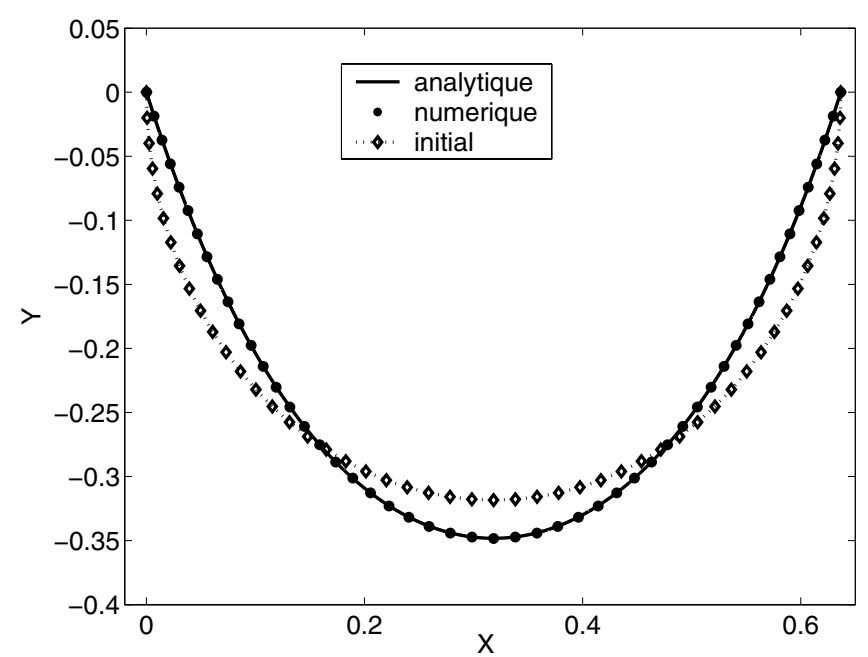

Fig. 1. Solutions numérique et analytique au problème 3D de la chaînette pour $N_{e}=50$.

voilier de $8 \mathrm{~m}$. Le câble a une longueur $L$ unité, une section $A=20 \mathrm{~mm}^{2}$ et un module d'Young $E=50000 \mathrm{MPa}$. Le chargement uniforme, porté par l'axe $\boldsymbol{y}$ est perpendiculaire à la ligne joignant les deux points fixes. La configuration initiale, dans le plan $O x z$, est un demi-cercle décomposé en 50 éléments linéaires (51 nœuds). Le coefficient de sub-relaxation $\zeta$ est fixé à $10^{-6}$, le critère d'arrêt $\epsilon=10^{-7}$ et $N=50000$.

Après deux millions d'itérations sur un PC $1.8 \mathrm{GHz}$ (trente secondes de calcul), la distance en chaque nœud entre les solutions analytique [10] et numérique est inférieure à un pour cent de la longueur du câble, ce qui valide notre modèle (Fig. 1).

\section{Modélisation numérique d'une voile et de son étai}

Dans cette section, nous décrivons brièvement le modèle de réseau de fils développé par Le Maître et al. [6] et nous mettons l'accent sur la méthode de couplage entre la voile et l'étai.

\subsection{Modèle du réseau de fils}

Le modèle du réseau de fils reprend le modèle du fil vu précédemment. C'est une extension qui va permettre d'approcher une structure bidimensionnelle sans épaisseur (une voile) à l'aide d'un ensemble de structure unidimensionnelle (le fil) [11]. Le réseau est constitué par l'enchevêtrement de deux familles de fils perpendiculaires, de module élastique linéique $K_{i}$, appelées trame indicée 1 et chaîne indicée ${ }_{2}$. Chaque nœud est repéré par ses coordonnées curvilignes $\left(a_{1}, a_{2}\right) \in \Omega_{m}$ auquel est associé le vecteur position $\underline{\boldsymbol{r}}\left(a_{1}, a_{2}\right) \in \mathcal{R}^{3}$ dans le repère absolu. La formulation variationnelle de l'équation du mouvement pour un élément de surface $\mathrm{d} a=\mathrm{d} a_{1} \mathrm{~d} a_{2} \in \Omega_{m}$ traduisant l'équilibre dynamique est établie pour des champs virtuels compatibles avec les liaisons (puissance de la liaison nulle) (Éqs. (3), (10)).

$$
\begin{aligned}
\iint_{\Omega_{m}}( & \left.\underline{\boldsymbol{T}}_{1} \cdot \frac{\mathrm{d} \underline{\boldsymbol{V}}^{*}}{\mathrm{~d} a_{1}}+\underline{\boldsymbol{T}}_{2} \cdot \frac{\mathrm{d} \underline{\boldsymbol{V}}^{*}}{\mathrm{~d} a_{2}}\right) \mathrm{d} a- \\
& \iint_{\Omega_{m}}\left(\underline{\boldsymbol{F}_{\boldsymbol{s}}}-\rho_{s} \underline{\ddot{\boldsymbol{r}}}\right) \cdot \underline{\boldsymbol{V}}^{*} \mathrm{~d} a-\int_{\Gamma} \underline{\boldsymbol{F}_{\boldsymbol{b}}} \cdot \underline{\boldsymbol{V}}^{*} \mathrm{~d} \Gamma=0
\end{aligned}
$$

Avec, pour $i=1,2$, les expressions de la tension et de la dilatation :

$$
\begin{array}{r}
\underline{\boldsymbol{T}}_{i}\left(a_{1}, a_{2}\right)=l_{i \perp} K_{i}\left(\alpha_{i}\left(a_{1}, a_{2}\right)-1\right) \underline{\boldsymbol{t}}_{i}\left(a_{1}, a_{2}\right) \geq 0 ; \\
\alpha_{i}\left(a_{1}, a_{2}\right)=\left\|\frac{\mathrm{d} \underline{\boldsymbol{r}}\left(a_{1}, a_{2}\right)}{\mathrm{d} a_{i}}\right\|
\end{array}
$$

$\underline{\boldsymbol{t}}_{i}\left(a_{1}, a_{2}\right)$ est le vecteur normal à la section du fil dans la direction $i, \boldsymbol{F}_{\boldsymbol{s}}\left(a_{1}, a_{2}\right) \in \mathcal{R}^{3}$ est le chargement extérieur appliqué sur $\Omega_{m}, \underline{\boldsymbol{F}_{\boldsymbol{b}}}\left(a_{1}, a_{2}\right) \in \mathcal{R}^{3}$ est le chargement extérieur appliqué sur la frontière $\partial \Omega_{m}$ notée $\Gamma$ si la frontière est libre (raccord entre deux laizes, chute de la voile...) $\Gamma_{0}$ dans le cas contraire et $\underline{\boldsymbol{\nu}}$ est le vecteur unitaire normal à la frontière $\Gamma$ et tangent à $\Omega_{m} \cdot \underline{\boldsymbol{V}}^{*}=0$ sur $\Gamma_{0}$. Nous avons la condition limite suivante sur $\Gamma$ :

$$
\begin{aligned}
& \nu_{1} \underline{\boldsymbol{T}}_{1}\left(a_{1}, a_{2}\right)+\nu_{2} \underline{\boldsymbol{T}}_{2}\left(a_{1}, a_{2}\right)=\underline{\boldsymbol{F}}_{\boldsymbol{b}}\left(a_{1}, a_{2}\right) ; \\
& \nu_{i}=\underline{\boldsymbol{\nu}} \cdot \frac{\underline{\boldsymbol{t}}_{i}\left(a_{1}, a_{2}\right)}{\left\|\underline{\boldsymbol{t}}_{i}\left(a_{1}, a_{2}\right)\right\|} \quad i=1,2
\end{aligned}
$$

La résolution numérique repose sur la méthode des éléments finis avec un schéma de Newmark pour les termes d'accélération. La formulation discrète du premier terme de l'équation d'équilibre (cf. Éq. (10)) basé sur le maillage $(i, j)$ de la voile (ou une partie de la voile : laize) à l'aide d'éléments triangle devient :

$$
\begin{array}{r}
\sum_{i=1}^{N_{i}} \sum_{j=1}^{N_{j}}\left[\left(\underline{\boldsymbol{T}}_{1}(i-1, j)-\underline{\boldsymbol{T}}_{1}(i, j)\right)+\left(\underline{\boldsymbol{T}}_{2}(i, j-1)\right.\right. \\
\left.\left.\left.-\underline{\boldsymbol{T}}_{2}(i, j)\right)\right] \cdot \underline{\boldsymbol{V}}^{*}(i, j)\right)
\end{array}
$$

avec la tension dans l'élément chaîne compris entre les nœuds $(i, j)$ et $(i+1, j)$ :

$$
\begin{aligned}
& \underline{\boldsymbol{T}}_{1}(i, j)=\left(l_{1 \perp}(i, j) K_{1}(i, j)\right. \\
& \left.\quad+l_{1 \perp}(i+1, j) K_{1}(i+1, j)\right)\left(\alpha_{1}(i, j)-1\right) \underline{\boldsymbol{t}}_{1}(i, j)
\end{aligned}
$$

Nous retrouvons le même système d'équations nonlinéaires résolu par la méthode itérative directe vue précédemment (Sect. 2.3).

La figure 2 présente la discrètisation d'un échantillon de tissu construit sur un maillage $(i, j)$. Les fils (traits continus) orthogonaux constituent la trame $(i)$ et la chaîne $(j)$. Nous avons placé sur cette figure les quatre tensions internes $-\underline{\boldsymbol{T}}_{1}(i, j),-\underline{\boldsymbol{T}}_{2}(i, j), \underline{\boldsymbol{T}}_{1}(i-1, j)$ et $\underline{\boldsymbol{T}}_{2}(i, j-1)$ qui s'appliquent au nœud $i, j$ équilibrant la force externe $\underline{\boldsymbol{F}}_{i, j}$. Ces tensions étant toujours tangentes aux directions principales (1 et ${ }_{2}$ ) du réseau de fils, ce 


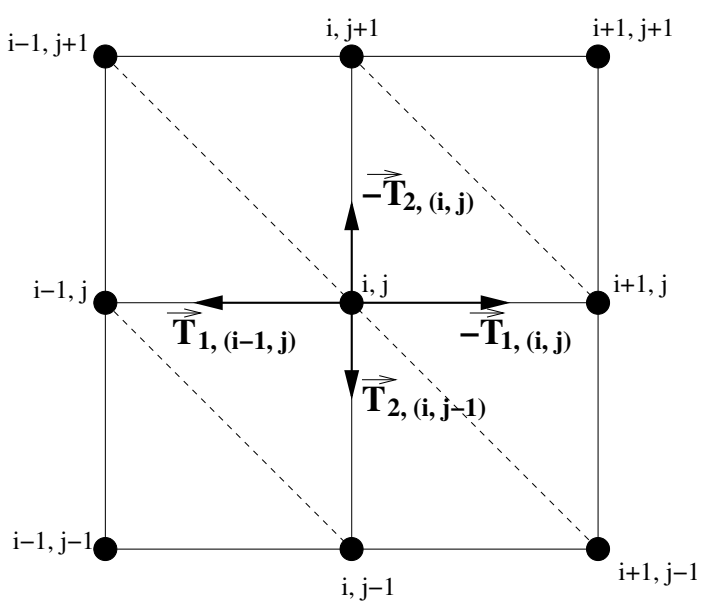

Fig. 2. Maillage et jeu de forces internes sur le nœud $i, j$.

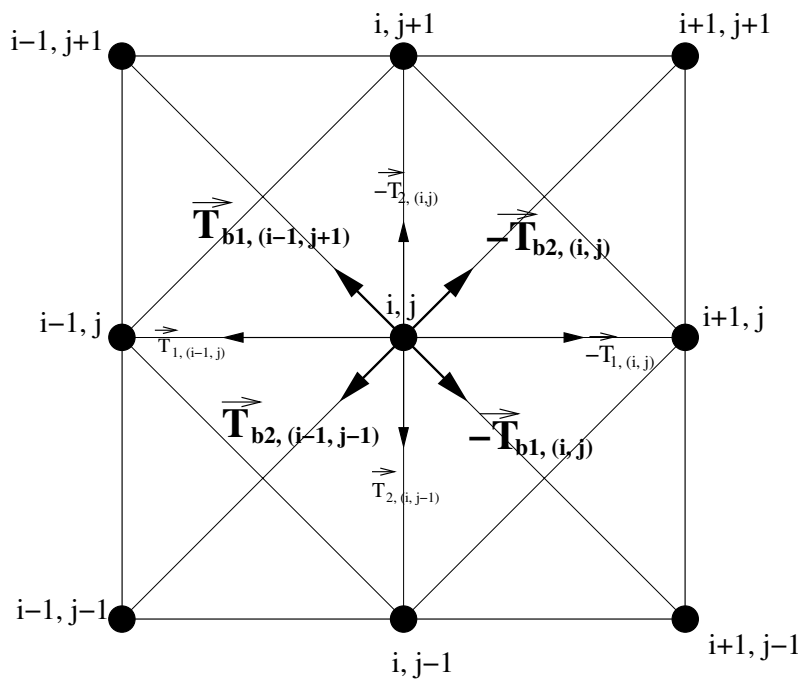

Fig. 3. Maillage et jeu de forces internes sur le nœud $i, j$ en prenant en compte la raideur dans le biais $\left(45^{\circ}\right)$ du tissu.

dernier ne présente aucune raideur dans le biais $\left(45^{\circ}\right)$. En considérant les mêmes équations discrètes et avec un maillage conservant le même nombre de nœuds mais faisant apparaître plus de triangles, il devient possible de prendre en compte le biais dans notre modèle. Sur la figure 3, nous remarquons alors que les forces internes précédentes se trouvent complétées par quatre autres tensions tangentes aux biais $-\underline{\boldsymbol{T}}_{b 1}(i, j),-\underline{\boldsymbol{T}}_{b 2}(i, j)$, $\underline{\boldsymbol{T}}_{b 1}(i-1, j+1)$ et $\underline{\boldsymbol{T}}_{b 2}(i-1, j-1)$.

\subsection{Modélisation des forces internes d'un tissu}

Le comportement mécanique des matériaux tissés utilisés pour la conception des voiles résulte essentiellement du caractère filaire de ces constituants. Dans l'équation (4) le module d'Young $E$ est le module d'élasticité homogène à une pression N.mm ${ }^{-2}$. L'extension à un réseau de fils n'est pas simplement la superposition de plusieurs fils. L'entrelacement des mèches, l'effet du tissage, la rigidité transverse, la

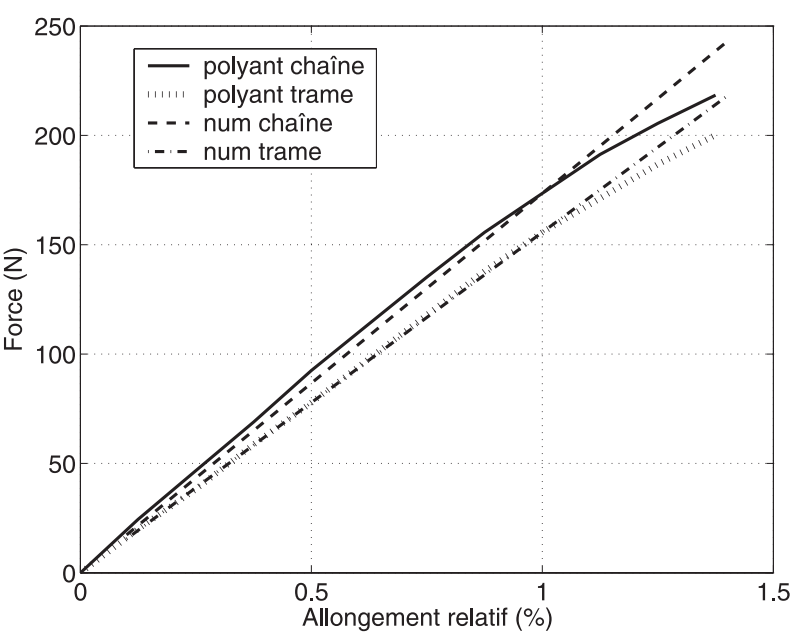

Fig. 4. Courbes de comportement numérique et expérimentale du PM05 (Polyant).

densité de mailles, confèrent aux tissus un comportement mécanique spécifique. Pour tenir compte du comportement mécanique du tissu, le module d'élasticité $K_{i}$ dans l'équation (11) est déterminé à partir d'essais de traction dans les directions de chaîne et trame sur une éprouvette rectangulaire $(2 \times 16$ inches $)$. Le module d'élasticité, exprimé en N.mm ${ }^{-1}$ est le rapport de la force de traction appliquée pour obtenir un allongement de $1 \%$ par la largeur de l'éprouvette (2 inches). Dans le calcul de la tension interne (cf. Éq. (11)) $K_{i}$ est multiplié par la longueur courante de l'élément fini perpendiculaire à la direction $i$. Sur la figure 4 nous présentons les résultats d'un essai de traction sur du PM05 fabriqué et commercialisé par Dimension-Polyant et d'un calcul réalisé sur une éprouvette discrétisée en 246 nœuds et 400 triangles. Le comportement linéaire décrit par le code numérique est proche des résultats expérimentaux pour des allongements inférieurs à $1 \%$. Ceci valide notre modèle car les déformations attendues pour une voile en navigation sont de l'ordre de 0 à $0,5 \%$.

\subsection{Couplage étai-voile}

Le guindant de la voile est relié à l'étai par une série de mousquetons qui peuvent coulisser le long de l'étai. Ils sont modélisés par des ressorts de longueur naturelle et de masse nulle et de raideur $K_{r_{i}}$. Ces ressorts, disposés en chaque nœud $i$ du guindant, vont exercer sur ceux-ci une force de rappel $\underline{\boldsymbol{F}}_{\boldsymbol{r}}$ produit de $K_{r_{i}}$ par une distance $d_{i}$ évoluant au cours du processus de déformation. $d_{i}$ est la distance entre les nœuds $i$ du guindant et de l'étai de position respective $\boldsymbol{r}_{\boldsymbol{g}_{i}}$ et ${\underline{\boldsymbol{r}_{i}}}_{i} \cdot{\underline{\boldsymbol{r}_{i}}}_{\boldsymbol{e}_{i}}$ est l'unique intersection entre le cercle de centre ${\underline{\boldsymbol{r}_{\boldsymbol{g}}}}_{i}$, de rayon $d_{i}$ et l'étai.

$$
d_{i}=\left\|\underline{\boldsymbol{r}}_{i}-\underline{\boldsymbol{r}}_{i}\right\|
$$


Cette force de rappel ${\underline{\boldsymbol{F}_{\boldsymbol{r}}}}_{i}$ est dirigée de manière à ramener le guindant vers l'étai.

$$
\underline{\boldsymbol{F}}_{i}=K_{r_{i}} d_{i} \frac{\underline{\boldsymbol{r}}_{i}-\underline{\boldsymbol{r}}_{i}}{\left\|\underline{\boldsymbol{r}}_{i}-\underline{\boldsymbol{r}}_{i}\right\|}
$$

${\underline{\boldsymbol{F}_{\boldsymbol{r}}}}_{i}$ correspond, d'une part, au chargement extérieur $\underline{\boldsymbol{F}_{\boldsymbol{b}}}\left(a_{1}, a_{2}\right)$ dans le modèle du réseau de fils représentant la voile (cf. Éq. (10)), et d'autre part, au chargement extérieur interpolé au nœud $i^{\prime}$ de l'étai à retrancher à $\underline{\boldsymbol{P}}_{i^{\prime}}$ dans le modèle du câble (cf. Éq. (7)). La raideur imposée des ressorts influe sur la distance que peut prendre le guindant vis-à-vis de l'étai. Les valeurs de $K_{r i}$ sont fixées telles que :

$$
d_{i}<0.01 L
$$

$L$ étant la longueur de l'étai.

\section{Modèle fluide : la méthode particulaire}

Nous présentons succinctement le modèle d'écoulement tridimensionnel de fluide parfait incompressible en régime instationnaire autour de profils minces développé par Rehbach [12]. La particularité de cette méthode réside, d'une part, dans le traitement lagrangien des particules tourbillonnaires constituant le sillage, et d'autre part, par la formulation du problème uniquement sur les frontières de l'écoulement ne nécessitant que le maillage des parois solides.

\section{1 Écoulement potentiel}

La décomposition de Helmholtz conduit à écrire le champ de vitesse absolu dans le repère relatif sous la forme :

$$
\underline{\mathbf{U}}_{a}(\underline{\boldsymbol{X}})=\underline{\mathbf{U}}_{\infty}+\underline{\nabla} \phi+\underline{\nabla} \wedge \underline{\boldsymbol{\psi}}
$$

Les potentiels $\phi$ et $\boldsymbol{\psi}$ traduisent respectivement l'influence des parois solides et de leurs sillages. La condition de glissement permet de calculer le saut de potentiel $[\phi]=\mu(s)$ représentant les parois (voiles).

$$
\begin{aligned}
\frac{1}{4 \pi} \iint_{\partial \mathcal{D}} \mu(s) & \underline{\nabla}\left(\frac{\left(\underline{\mathbf{x}}\left(s^{\prime}\right)-\underline{\mathbf{X}}(s)\right) \cdot \underline{\mathbf{n}}(\mathbf{\mathbf { X }}(s))}{\left\|\underline{\mathbf{X}}\left(s^{\prime}\right)-\underline{\mathbf{x}}(s)\right\|^{3}}\right) \cdot \underline{\mathbf{n}}\left(s^{\prime}\right) \mathrm{d} s \\
=-\left(\underline{\mathbf{U}}_{\infty}+\underline{\nabla}\right. & \left.\wedge \underline{\boldsymbol{\psi}}\left(s^{\prime}\right)-\underline{\mathbf{U}}_{e}\left(s^{\prime}\right)\right) \cdot \underline{\mathbf{n}}\left(s^{\prime}\right)
\end{aligned}
$$

Avec $\underline{\mathbf{X}}(s)$ et $\underline{\mathbf{X}}\left(s^{\prime}\right)$ deux points de la surface $\partial \mathcal{D}$ et $\underline{\mathbf{U}} e_{e}\left(s^{\prime}\right)$ la vitesse d'entraînement du corps intégrant le mouvement de corps solide (moment du torseur cinématique d'entraînement) et la vitesse de déformation de la structure $\left(\underline{\dot{\mathbf{X}}}\left(s^{\prime}\right)\right)$. La solution $\mu(s)$ de ce problème conduit à résoudre un système linéaire en discrètisant les parois en $N_{f}$ facettes quadrangulaires planes. Le champ de vitesse en tout point $\underline{\mathbf{Y}}$ induit par le saut de potentiel est donné par la relation intégrale suivante :

$$
\underline{\mathbf{U}}_{\phi}(\underline{\mathbf{Y}})=\underline{\nabla} \phi(s)=-\frac{1}{4 \pi} \iint_{\partial \mathcal{D}} \mu(s) \underline{\nabla}\left(\frac{(\underline{\mathbf{Y}}-\underline{\mathbf{X}}(s)) \cdot \underline{\mathbf{n}}(\underline{\mathbf{X}}(s))}{\|\underline{\underline{X}}-\underline{\mathbf{X}}(s)\|^{3}}\right) \mathrm{d} s
$$

\subsection{Sillage}

La circulation totale dans le fluide étant conservée, toute variation de la circulation autour de l'obstacle est nécessairement compensée par une émission de vorticité dans le sillage dont on impose l'échappement au bord de fuite ce qui exclut les situations de décrochage. Les relations donnant la position $\underline{\mathbf{X}}_{i r}$ et le poids $\underline{\boldsymbol{\Omega}}_{i a}$ de chaque particule tourbillonnaire $i$ à l'instant de leur création, permettent de définir le transfert de vorticité de la couche limite vers l'écoulement extérieur.

$$
\left\{\begin{aligned}
\underline{\boldsymbol{\Omega}}_{i a}= & {\left[\delta l_{i}\left(\mu_{i}(t+\Delta t)-\mu_{i}(t)\right)\right] \underline{\mathbf{i}} } \\
& +\left[\Delta t\left|\underline{\mathbf{U}}_{b f}-\underline{\mathbf{U}}_{e}\right| \frac{\mu_{i+1}-\mu_{i-1}}{2}\right] \underline{\mathbf{j}} \\
\underline{\mathbf{x}}_{i r}= & \underline{\mathbf{X}}_{b f}+\left(\underline{\mathbf{U}}_{e}+\underline{\mathbf{U}}_{b f}\right) \frac{\Delta t}{2}
\end{aligned}\right.
$$

avec $\left(\underline{\mathbf{X}}_{b f}, \underline{\mathbf{i}}, \underline{\mathbf{j}}, \underline{\mathbf{n}}_{i}\right)$ le repère local au bord de fuite ( $\underline{\mathbf{i}}$ tangent au bor $\bar{d}$ de fuite), $\underline{\mathbf{U}}_{b f}$ la vitesse moyenne intrados/extrados de l'écoulement au bord de fuite et $\delta l_{i}$ la longueur du segment émetteur.

La forme discrète du système caractérisant l'évolution dans le fluide d'une particule de position $\underline{\mathbf{X}}_{i r}$, et portant un tourbillon $\underline{\boldsymbol{\Omega}}_{i a}$ devient :

$$
\left\{\begin{aligned}
\frac{D \underline{\mathbf{X}}_{i r}}{D t}= & \left(\underline{\mathbf{U}}_{\omega}+\underline{\mathbf{U}}_{\phi}+\underline{\mathbf{U}}_{\infty}-\underline{\mathbf{U}}_{e}\right)\left(\underline{\mathbf{X}}_{i r}, t\right) \\
\frac{D \underline{\boldsymbol{\Omega}}_{i a}}{D t}= & -\left(\underline{\boldsymbol{\Omega}}_{i a} \cdot \underline{\nabla}\right)\left(\underline{\mathbf{U}}_{\omega}+\underline{\mathbf{U}}_{\phi}\right. \\
& \left.+\underline{\mathbf{U}}_{\infty}-\underline{\mathbf{U}}_{e}\right)\left(\underline{\mathbf{X}}_{i r}, t\right)+\left(\underline{\mathbf{\Phi}} \wedge \underline{\boldsymbol{\Omega}}_{i a}\right)
\end{aligned}\right.
$$

Avec $\underline{\Phi}$ la résultante du torseur cinématique d'entraînement, $\frac{D}{D t}=\frac{\partial}{\partial t}+\left(\underline{\mathbf{U}}_{r} \cdot \underline{\nabla}\right)$ la dérivée particulaire dans le repère relatif et $\underline{\mathbf{U}}_{\omega}=\underline{\nabla} \wedge \underline{\boldsymbol{\psi}}$ obtenu au moyen de la loi de Biot et Savart.

$$
\underline{\mathbf{U}}_{\omega}(Y)=-\frac{1}{4 \pi} \iiint_{\mathcal{D}} \frac{\underline{\boldsymbol{\omega}}\left(Y^{\prime}\right) \wedge \underline{\mathbf{Y} \mathbf{Y}^{\prime}}}{\left|\underline{\mathbf{Y} \mathbf{Y}^{\prime}}\right|^{3}} \mathrm{~d} v\left(Y^{\prime}\right)
$$

Le comportement singulier de ces équations lorsque $\underline{\mathbf{Y Y}^{\prime}} \rightarrow 0$ conduit à introduire une fonction de regularisation $[13]$

\subsection{Efforts exercés par le fluide}

Le calcul des efforts au point de contrôle $P_{k}$ de chaque facette $k$ de surface $S_{k}$ s'effectue à partir de la loi de Joukovski :

$$
\underline{\mathbf{F}}_{k}=S_{k} \frac{\partial \mu_{k}}{\partial t} \underline{\mathbf{n}}_{k}+\sum_{i=1}^{4} v_{i} \underline{\mathbf{U}}_{i} \wedge \underline{\boldsymbol{\Gamma}}_{i}
$$

Avec $\underline{\mathbf{U}}_{i}$ la vitesse au milieu du segment $i, \underline{\boldsymbol{\Gamma}}_{i}$ l'intensité du segment tourbillonnaire portée par le côté $i$ après équivalence doublet-tourbillon (Hess \& Smith) et $v_{i}$ est un coefficient de pondération fonction de la distance du milieu du segment $i$ considéré au point de contrôle $P_{k}$ si le segment appartient à deux facettes.

Une série de tests comparatifs numérique/expérience (en soufflerie) a été effectuée sur des voiles indéformables afin de valider la modélisation fluide [14]. 


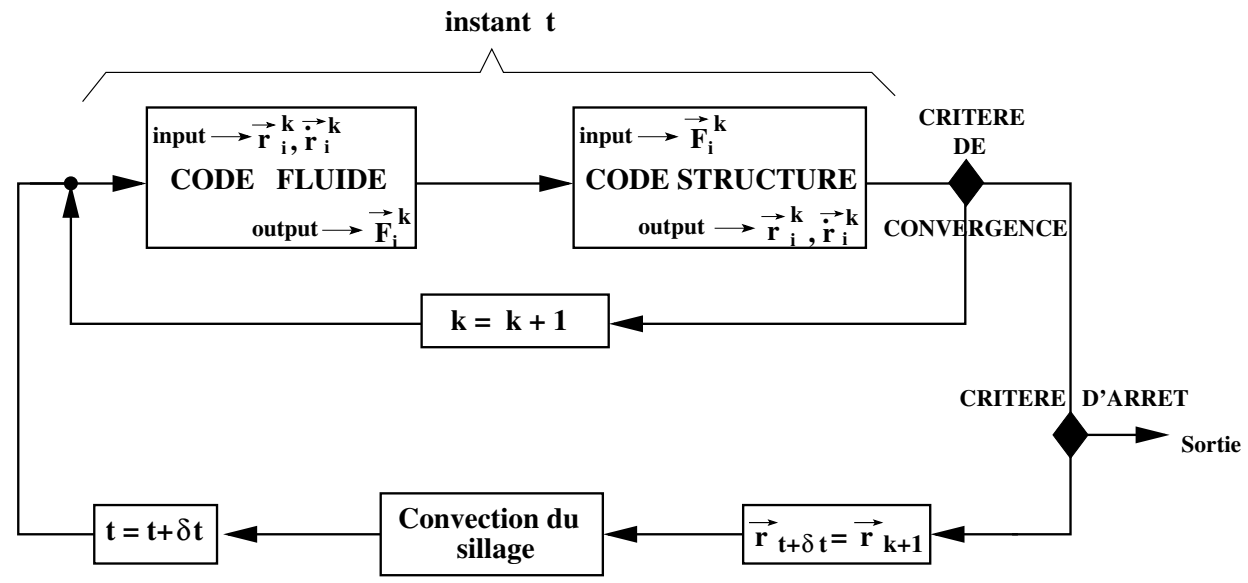

Fig. 5. Schéma du couplage fluide structure.

\section{Couplage fluide structure}

L'écoulement et la structure interagissent fortement : l'écoulement dépend de la forme de la structure qui elle-même dépend des efforts exercés par l'écoulement à laquelle elle est soumise. Cette interaction est exprimée à l'aide des équations de couplage qui sont de nature cinématique (continuité de la vitesse normale des milieux fluide et solide sur l'interface dans sa configuration déformée) et dynamique (égalité de l'effort extérieur exercé sur la structure et de l'effort de pression due au fluide). Le problème complet s'écrit sous la forme d'un ensemble d'équations non-linéaires qui peuvent être intégrées dans le temps par une méthode itérative suivant le schéma représenté sur la figure 5 . La boucle interne est pilotée par un critère de convergence sur la stationnarité de la géométrie $\underline{\boldsymbol{r}}_{i}^{k}$ soumis au chargement $\underline{\boldsymbol{F}}_{i}^{k}$. La boucle externe permet d'appréhender des effets dynamiques. Le critère d'arrêt peut être la convergence du processus itératif de résolution des équations du mouvement en régime stationnaire (équilibre du voilier).

\subsection{Résultats}

La voile utilisée est un génois d'un bateau de $8 \mathrm{~m}$ avec $8,85 \mathrm{~m}$ de longueur de guindant, 4,3 $\mathrm{m}$ de bordure et $8,40 \mathrm{~m}$ de chute, pour une surface totale de $18 \mathrm{~m}^{2}$. La voile, initialement à coupe orientée bi-radiale, a été simplifiée (laizes horizontales) pour des raisons de correspondance de maillage entre les problèmes fluide et structure, Hauville et al. (2003) [9]. Nous considérons un écoulement incident uniforme $A W S=10 \mathrm{kt}$ sous une incidence $A W A=24^{\circ}$. L'équilibre du bateau n'est pas calculé; le mât porté par l'axe $\boldsymbol{y}$ reste perpendiculaire à la surface libre (gîte nulle). Afin de montrer l'influence de la déformation de l'étai sur le volume de la voile (donc sur les performances du bateau) deux calculs aéroélastiques ont été réalisés en considérant l'étai fixe ou non. Pour l'ensemble des calculs, les longueurs de drisse et d'écoute sont constantes, la position du point d'amure et du roller sont fixes. Dans le cas de l'étai fixe, aucun déplacement

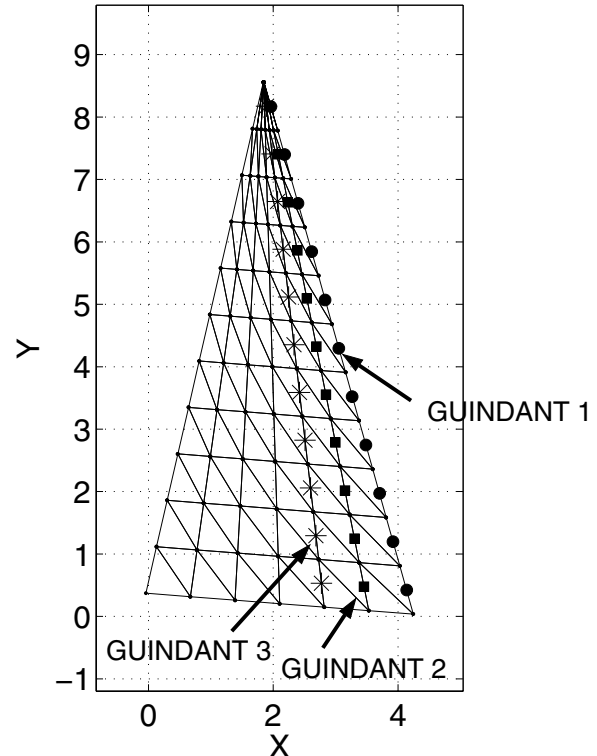

Fig. 6. Maillage dans le plan $O x y$ et position des tourbillons de bord d'attaque.

n'est autorisé pour le point de drisse. Le point d'écoute peut se déplacer quelle que soit la configuration de l'étai. La position du roller et le point d'écoute sont définis de façon à régler au mieux le bord d'attaque de la voile. Dans la pratique, une voile est bien réglée lorsque l'écoulement au bord d'attaque de la voile est laminaire et qu'il reste attaché tout le long de la surface portante. Dans notre modèle, cela peut se traduire, en partie, par une composante nulle du vecteur tourbillon porté par les particules fixes distribuées sur les segments constituant le bord d'attaque de la voile. Nous nous sommes intéressés à la valeur de cette composante sur une première envergure constituée par le bord d'attaque mais aussi deux autres envergures consécutives comme illustré sur la figure 6 .

La figure 7 montre la valeur de cette composante portée par les segments pour ces trois envergures. La voile avec étai fixe a été réglée de façon à annuler au mieux l'intensité des tourbillons de bord d'attaque. Ces mêmes 


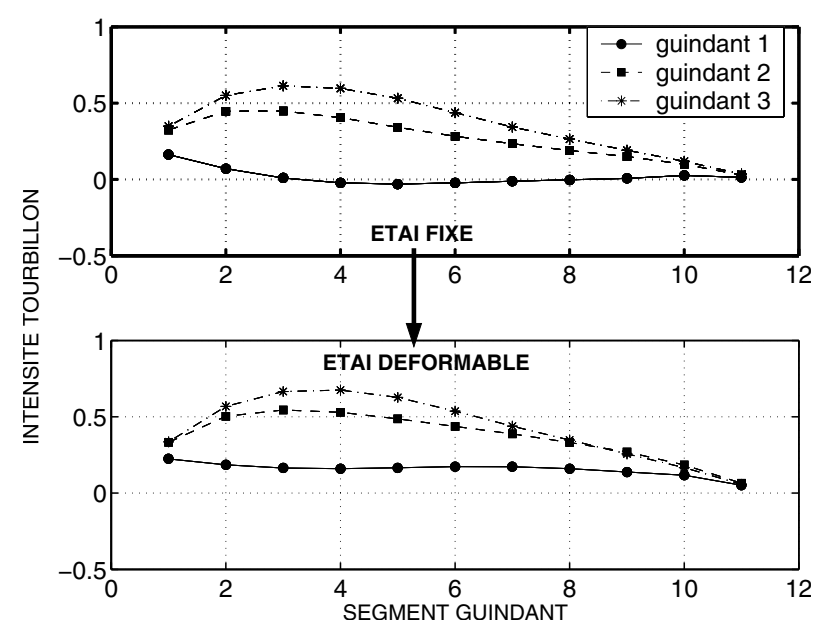

Fig. 7. Intensité des tourbillons de bord d'attaque selon $O y$.

réglages ont été appliqués au cas de la voile avec un étai déformable. Les tourbillons de bord d'attaque ne sont plus nuls, mais positifs ce qui signifie que le bord d'attaque s'est affiné. Le profil au niveau du bord d'attaque, c'està-dire au niveau de son premier quart de corde, possède moins de creux.

La figure 8 montre le volume de la voile (suivant $\underline{\boldsymbol{z}}$ ) après convergence des calculs. Les modules élastiques $K_{i}$, volontairement faibles, sont identiques dans les directions de chaîne et trame pour accentuer la déformation de la voile. Le volume de la voile avec l'étai libre est $20 \%$ supérieur qu'avec l'étai fixe. La figure 8 montrant les déformées dans le plan $O x z$ permet de remarquer un léger recul du creux sur l'arrière.

La figure 9 montre quant à elle la prise de flèche de l'étai dans le plan $O x z$, ainsi que le changement de géométrie de la chute qui s'ouvre plus en bas et se referme légèrement dans le haut, ce qui concorde avec le recul du creux.

Sur la figure 10, on remarque l'évolution des composantes dans le plan Oxyz de la force vélique adimensionnelle. La variation de la valeur des composantes est un paramètre permettant de juger de la convergence du calcul et de l'état d'équilibre du système. Les composantes $F z$ et $F x$ sont, dans le cas d'un étai déformable, respectivement $8 \%$ et $20 \%$ supérieures par rapport au cas d'une voile avec un étai fixe. Au final, l'intensité de la force propulsive, correspondant à l'effort aérodynamique projeté sur l'axe du bateau ( $\underline{\boldsymbol{x}})$, augmente de $20 \%$. Toutefois, ces comparaisons de force propulsive sont à relativiser. D'une part, les calculs se sont faits à gîte fixe. La contribution de la composante $F z$ est donc à relativiser car son augmentation entraîne une gîte plus importante, soit une modification de l'écoulement autour de la voile. D'autre part, le modèle « fluide parfait » que nous utilisons pour calculer l'écoulement fluide, ne modélise pas les décollements de couche limite. Par conséquent l'écoulement est toujours attaché. La portance de la voile peut donc se voir augmentée par le modèle, alors que dans la réalité un décollement de couche limite entraîne une chute de portance.
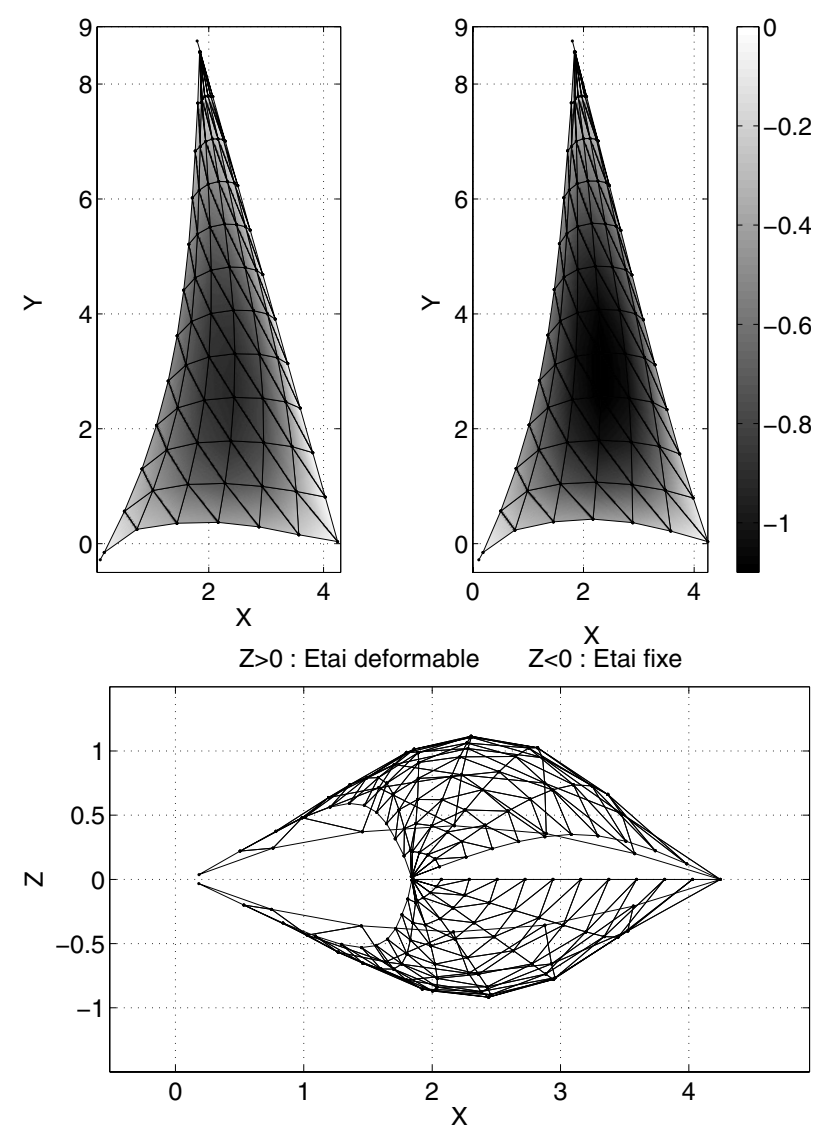

Fig. 8. Déformation de la voile avec étai libre et fixe dans les plans $O x y$ (vue de coté gauche : étai fixe, droit : étai libre) et Oxz (vue de dessus).

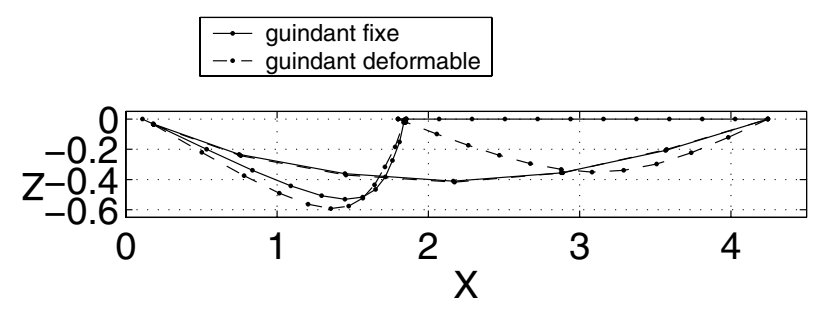

Fig. 9. Frontières de la voile dans le plan $O x z$, dans le cas d'un étai fixe et d'un étai déformable.

\section{Conclusions}

Le modèle aéroélastique présenté permet d'étudier l'interaction d'une structure tridimensionnelle idéalement flexible, décrit par un système de réseau de fils, avec un écoulement aérodynamique instationnaire, modélisé par une méthode de surface portante associé à un sillage particulaire. La méthode utilisée permet de prendre en compte la déformation de l'étai, modélisé par un fil, dans le calcul aéroélastique. Les résultats obtenus à travers les différentes simulations aéroélastiques, ont montré l'importance de la flèche d'étai dans les performances du voilier. Le temps de calcul pour la convergence du modèle est globalement proportionnel au module élastique. Ainsi, la modélisation réelle d'une voile et de ses renforts, caractérisés par l'augmentation locale du module élastique, 

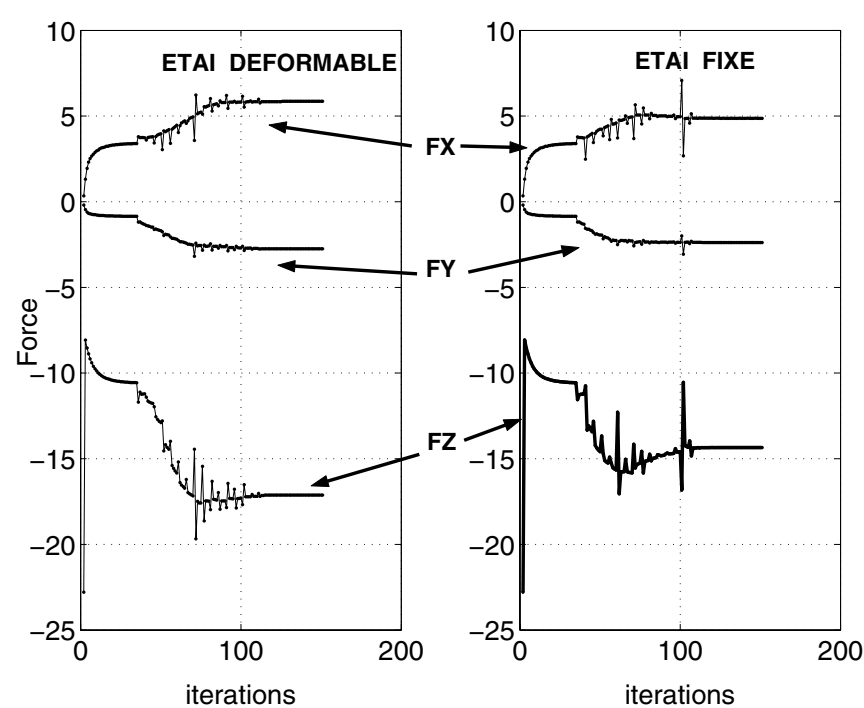

Fig. 10. Composante de la force vélique dans le cas de voiles avec étai fixe et étai déformable.

nécessitant un coefficient de sous-relaxation adapté dans ces zones, accroît notablement les temps de calcul. Le modèle sera prochainement optimisé par la prise en compte, d'une part, d'éléments rigidifiants (lattes) dans la structure souple, et d'autre part, du gréement, par le couplage d'un modèle de câble en petites déformations et grands déplacements au modèle aéroélastique actuel.

\section{Références}

[1] Y. Roux, S. Huberson, F. Hauville, J.P. Boin, M. Guilbaud, M. and Ba, Yacht performance prediction: Towards a numerical vpp, in: High Performance Yacht Design Conference, December 2002, Auckland

[2] O. Le Maître, Contribution numérique à la résolution de problèmes d'interaction fluide structure, Thèse, Institut de mécanique INSA de Rouen, France, 1998
[3] T. Charvet, F. Hauville, S. Huberson, Numerical simulation of the flow over sails in real sailing conditions, J. Wind Eng. Ind. Aerod. Special Issue Sail Aerodynamics 63 (1996) 111-129

[4] T. Charvet, Résolution numérique de problèmes liés au comportement des voiles de bateau, Thèse, École Polytechnique, LIMSI-CNRS, France, 1992

[5] F. Muttin, Modélisation de membranes. Application à l'analyse mécanique des voiles de bateau, Thèse, Université de Nice Sophia Antipolis, 1989

[6] O. Le Maître, S. Huberson, J.E. Souza De Cursi, Application of a non-convex model of fabric deformations to sail cut analysis, J. Wind Eng. Ind. Aerod. 63 (1996) $77-93$.

[7] J. Valette, Simulation du comportement mécanique d'un ensemble voiles gréement de bateau, Thèse, Université d'Orléans, France, 2001

[8] P. Heppel, Accuracy in sail simulation: Wrinkling and growing fast sails, in: High Performance Yacht Design Conference, Auckland, 2002

[9] F. Hauville, Y. Roux, Réglage dynamique d'une voile par une méthode d'interaction fluide-structure, in : $9^{\text {es }}$ Journées de l'Hydrodynamique, Poitiers, France, ISSN 1161-1847, 2003, pp. 221-234

[10] F. Axisa, Modélisation des systèmes mécaniques : systèmes continus, Hermès sciences publication, 22001

[11] O. Le Maître, J.E. Souza De Cursi, S. Huberson, Large displacements analysis for ideally flexible sails, Eur. J. Mech. A-Solid. 17 (1998) 619-636

[12] C. Rehbach, Numerical calculation of three dimensional unsteady flows with vortex sheets, AIAA, 16th Huntsville, 1978, paper 1978-111

[13] J.T. Beale, A. Majda, High order accurate vortex methods with explicit velocity kernels, J. Comput. Phys. 58 (1985) 188-208

[14] T. Charvet, S. Huberson, Numerical calculation of the flow around sails, Eur. J. Mech. B-Fluids 11 (1992) 599610

[15] J.E. Souza De Cursi, Stress unilateral analysis of mooring cables, Int. J. Numer. Meth. Eng. 34 (1992) 279-302

$\overline{\text { Retrouvez nos articles sur le site : }}$ www.edpsciences.org/meca 